\title{
Exercício de fortalecimento para o manejo de tendinopatia do manguito rotador
}

\author{
Strengthening exercise for the management of rotator cuff tendinopathy \\ Ejercicio de fortalecimiento para el manejo de la tendinopatía del manguito rotador
}

Recebido: 25/10/2021 | Revisado: 02/11/2021 | Aceito: 08/11/2021 | Publicado: 13/11/2021

Kathleen Kátia Banhos Henrique
ORCID: https://orcid.org/0000-0002-1982-8276
Centro Universitário da Amazônia, Brasil
E-mail: kathleenhenrique1 @ gmail.com
David Luna de Jesus
ORCID: https://orcid.org/0000-0002-0973-0929
Centro Universitário da Amazônia, Brasil
E-mail: daviluna5517@gmail.com
Amanda Maria Alvarenga Bahia
ORCID: https://orcid.org/0000-0002-1955-4463
Centro Universitário da Amazônia, Brasil
E-mail: amandaalvarenga18@ hotmail.com
Wanderson Fernandes Silva
ORCID: https://orcid.org/0000-0001-9389-5795
Centro Universitário da Amazônia, Brasil
E-mail: wandersonfsm @ outlook.com

\section{Resumo}

O manguito rotador RC é formado pelos tendões supraespinhal, subescapular, infra espinal e redondo menor. Sendo que todos os tendões tem origem na parte anterior e superior e parte posterior da região do ombro. A estrutura do tendão é formada por seis a nove feixes estruturalmente independentes, e por água e colágeno do tipo I. Objetivo: O objetivo do presente estudo foi determinar se o fortalecimento muscular é eficaz em Tendinopatia do manguito rotador. Métodos: A revisão de literatura foi realizada nas bases de dados virtuais Pubmed e PEDro. Os descritores utilizados foram os seguintes: Exercícios de fortalecimento; fisioterapia; tendinopatia do manguito rotador. Os estudos deveriam obedecer aos seguintes critérios de elegibilidade: artigos publicados na língua inglesa, intervalo de publicação de cinco anos, entre 2017 a 2021, ensaios clínicos randomizados, com pontuação superior a 6 na escala de qualidade PEDro. Resultados: Foram achados 96 artigos, sendo que entraram apenas 7 artigos relacionados ao tema. Conclusão: O presente estudo de revisão bibliográfica verificou a eficácia do fortalecimento muscular na Tendinopatia RC, em todos os estudos inclusos, os exercícios de FM excêntricos e alongamentos obtiveram grandes resultados, desde o período do quadro álgico até a funcionalidade e total mobilidade de cada participante, porém é de sumo importância obter novos estudos afim de traçar novos meios de tratamento da tendinopatia no manguito rotador. Palavras-chave: Exercícios de fortalecimento; Fisioterapia; Tendinopatia do manguito rotador.

\begin{abstract}
The RC rotator cuff is formed by the supraspinatus, subscapularis, infraspinatus, and teres minor tendons. Since all tendons originate from the anterior and superior part and the posterior part of the shoulder region. The tendon structure is formed by six to nine structurally independent bundles, and by water and type I collagen. Objective: The aim of the present study was to determine whether muscle strengthening is effective in rotator cuff tendinopathy. Methods: The literature review was carried out in the virtual databases Pubmed and PEDro. The descriptors used were the following: Strengthening exercises; physiotherapy; Rotator cuff tendinopathy. Studies should meet the following eligibility criteria: articles published in English, publication interval of five years, between 2017 and 2021 , randomized clinical trials, with a score greater than 6 on the PEDro quality scale. Results: 96 articles were found, with only 7 articles related to the topic. Conclusion: This literature review study verified the effectiveness of muscle strengthening in CR Tendinopathy, in all included studies, eccentric FM exercises and stretching obtained great results, from the period of pain to functionality and total mobility of each participant, however, it is extremely important to obtain further studies in order to outline new ways of treating tendinopathy in the rotator cuff.
\end{abstract}

Keywords: Strengthening exercises; Physiotherapy; Rotator cuff tendinopathy.

\section{Resumen}

El manguito rotador RC está formado por los tendones supraespinoso, subescapular, infraespinoso y redondo menor. Dado que todos los tendones se originan en la parte anterior y superior y en la parte posterior de la región del hombro. La estructura del tendón está formada por seis a nueve haces estructuralmente independientes, y por agua y colágeno tipo I. Objetivo: El objetivo del presente estudio fue determinar si el fortalecimiento muscular es efectivo en la 
tendinopatía del manguito rotador. Métodos: La revisión de la literatura se realizó en las bases de datos virtuales Pubmed y PEDro. Los descriptores utilizados fueron los siguientes: Ejercicios de fortalecimiento; fisioterapia; Tendinopatía del manguito rotador. Los estudios deben cumplir con los siguientes criterios de elegibilidad: artículos publicados en inglés, intervalo de publicación de cinco años, entre 2017 y 2021, ensayos clínicos aleatorizados, con una puntuación superior a 6 en la escala de calidad PEDro. Resultados: se encontraron 96 artículos, con solo 7 artículos relacionados con el tema. Conclusión: Este estudio de revisión de la literatura verificó la efectividad del fortalecimiento muscular en la Tendinopatía CR, en todos los estudios incluidos los ejercicios excéntricos de FM y elongación obtuvieron grandes resultados, desde el período de dolor hasta la funcionalidad y movilidad total de cada participante, sin embargo, es sumamente importante obtener más estudios con el fin de delinear nuevas formas de tratamiento de la tendinopatía en el manguito rotador.

Palabras clave: Ejercicios de fortalecimiento; Fisioterapia; Tendinopatía del manguito rotador.

\section{Introdução}

O manguito rotador é formado pelos tendões supraespinhal, subescapular, infra espinal e redondo menor. Todos os tendões tem origem na parte anterior e superior e parte posterior da região do ombro. A estrutura do tendão é formada por seis a nove feixes estruturalmente independentes, e por água e colágeno do tipo I. Segundo Lewis et al. (2014) “O tecido normal do tendão sofre remodelação contínua (degradação e reconstrução)”.

O autor Lewis et al. (2014), relata que a "Tendinopatia é um termo genérico sem implicações etiológicas, bioquímicas ou histológicas e é usado para descrever a patologia e a dor decorrente de um tendão. As causas podem ser intrínsecas ou extrínsecas". As Intrínsecas são as inflamações dentro do tendão que geralmente são causadas por causa da sobrecarga ou movimentos repetitivos, já a extrínsecas é uma irritação geralmente causa pelo acrômio, que se não obtiver resultado no tratamento com medicamentos e fisioterapia tem que fazer a cirurgia. (Lewis et al., 2014).

A tendinopatia do Manguito rotador são facilmente identificados pela dor, perda de função e fraqueza dos músculos do manguito rotador (RC). É a terceira doença que mais afeta a população com $16 \%$, sendo as dores nas costas em primeiro lugar e em segundo dor no joelho. Segundo Lewis et al, (2014) "A incidência de dor no ombro aumenta substancialmente com a idade e, para pessoas com mais de 65 anos, a dor no ombro é o problema musculoesquelético mais comum.”, ou seja, o ombro é o membro mais utilizado para realizar atividades cotidianas, e de produtividade no trabalho, movimentos repetitivos e de grande sobrecarga podem vim a gerar uma RC.

Longo et al., (2011) “A doença do manguito rotador é particularmente comum na população trabalhadora, causando altos custos de saúde nos países industrializados ocidentais e custos relevantes para os sistemas de compensação dos trabalhadores. Os dados epidemiológicos sobre a associação entre o uso excessivo mecânico e o rompimento do manguito são inconclusivos. A taxa de prevalência da síndrome do manguito rotador em trabalhadores varia entre $29 \%$ naqueles altamente expostos ao trabalho repetitivo e $16 \%$ naqueles fracamente expostos ao trabalho repetitivo".

Os fatores de riscos estão associados a idade, diabetes miellitus. Titchener et al. (2014) "sugere uma tendinose em vários locais, incluindo epicondilite lateral e medial, síndrome do túnel do carpo, doença de Quervain e dedo em gatilho;” estes estão correlacionados na literatura com alguns possíveis fatores de risco .A Diabetes tem que ter a insulina ou outro medicamento no auxílio do manguito rotador, A idade é associada a pacientes idosos com degeneração pela idade, epicondilite lateral, medial, dedo em gatilho, síndrome do túnel do carpo e doença de Quervain estão relacionadas por causa da carga. (Titchener et al, 2014).

O diagnóstico clinico é realizado através de exames de imagens com a ressonância magnética que irá mostrar se houve alguma ruptura dos tendões, degeneração e inflamação e consequentemente exames laboratoriais. A sensibilidade, dor e a perda de função são os principais sintomas. LEWIS et al. (2014) "testes clínicos são baseados em testes de referência padrão ouro que identificaram falha estrutural [..]”, esses achados podem validar o diagnostico ou invalidar, podendo assim realizar investigações como padrão ouro com uma US, ressonância magnética e observação direta durante a cirurgia. 
De acordo com o autor Lewis, (2009) "Exemplos de testes clínicos usados para identificar a patologia estrutural em uso atual incluem o teste de compressão ativa O'Brien para patologia labial superior, o teste de comprimento da cápsula posterior para avaliar a extensibilidade da cápsula glenoumeral posterior e o " teste supraespinhal " de Jobe para avaliar a força e a resposta à dor da unidade musculotendinosa supraespinhal. Outros testes incluem o sinal de Neer, que foi adotado com outros testes, como o teste de Hawkins e o Teste de esforço de resistência à rotação interna, métodos clínicos para implicar o acrômio como a causa dos sintomas apresentados no ombro".

$\mathrm{Na}$ fisioterapia foram abordados vários protocolos de tratamento com exercícios em cadeia aberta, fechada, isométricos e alongamentos. Alongamentos foram realizados antes de todos os exercícios de FM, exercício isométricos com membro afetado e não afetado, cadeia aberta e fechada com auxílios de faixas elásticas e alteres, ambos os protocolos obtiveram resultados positivos quanto a melhora da função e da dor. Objetivo do presente estudo foi determinar se o fortalecimento muscular é eficaz em manguito rotador. (Boudreau et al, 2019).

De acordo com Littlewood et al., (2012) "A patologia da tendinopatia do manguito rotador demonstrou demonstrar alterações patológicas semelhantes aos distúrbios do tendão em outras áreas do corpo, por exemplo, o cotovelo, onde o exercício carregado (contra a gravidade ou resistência) mostrou resultados benéficos. Portanto, parece plausível que exercícios intensos também possam ter um papel a desempenhar no manejo desses transtornos".

\section{Metodologia}

\section{Design de estudo}

A produção deste estudo de revisão sistemática da literatura foi montado de acordo com as recomendações do PRISMA (a declaração PRISMA tem o propósito de ajudar escritores a se aperfeiçoar em meta-analises e relato de revisões sistemáticas). Portanto, três investigadores, foram prudentes quanto a coleta de informações nas bases de dados, qualquer tipo de intercorrência no grupo, era solucionada através de diálogo em encontros subsequente. (Moher et al., 2009).

\section{Mecanismo de estudo}

O recente estudo buscou responder ao dilema traçado em: Exercício de Fortalecimento para o manejo de Tendinopatia do Manguito Rotador (MR) valida eficácias positivas? Para a estrutura desta revisão foi feita uma análise bibliográfica por meio de plataformas digitais: Pubmed e outras Plataformas associadas a Pubmed. A qualidade dos estudos foi avaliada na escala PEDro, e analisado pelos pesquisadores com o intuito de tirar dúvidas. Os unitermos utilizados na atual pesquisa foram: Exercícios de fortalecimento; fisioterapia; tendinopatia do manguito rotador. A busca de matérias perdurou de Janeiro até Outubro de 2021.

\section{Critérios de inclusão e exclusão}

Para estruturar o presente estudo de revisão sistemática, os artigos necessitariam cumprir com os respectivos critérios de elegibilidade: busca de estudos divulgados somente na língua inglesa, que se tornaram públicos entre os anos de 2016 a 2021, estudos pilotos, ensaios clínicos randomizados, atendendo uma pontuação superior que 7 na escala de qualidade PEDro, e que contivessem o tratamento da tendinopatia do manguito rotador através de exercícios de fortalecimento muscular. Foram excluídos artigos de estudos de caso, revisão de literatura, comentário clinico, estudo de coorte, estudos que obtivessem outras afecções de ombro, pesquisa clínica, estudo comparativo.

3. Variáveis Analisadas 
Para compor o presente estudo foram analisados os seguintes fatores como; estudos recentes e tais autores, tipos de intervenções fisioterapêuticas e frequência dos tratamentos, amostras com diferentes grupos, resultados e conclusões dos seguintes estudos, e avaliação metodológica segundo a escala PEDro.

\section{Escala Pedro}

O presente estudo utilizou-se da escala PEDro, visando obter a melhor qualidade metodológica de estudos publicados, a referida escala utiliza de 11 critérios.

No critério 1 refere-se a elegibilidade, e clareza da descrição em seus critérios de inclusão e exclusão. No critério 2 se diz respeito a divisão dos participantes se foram realizadas em grupos randomizados. Já no critério 3, os participantes têm que ser alocados secretamente nos grupos sem que ninguém saiba de cada alocação, até mesmo o Fisioterapeuta. O critério 4 se trata das variáveis prognósticos e a homogeneidade no processo de intervenção do tratamento. $\mathrm{O}$ critério 5 cita se o participante realizou de forma cega o tratamento, sem saber o tipo de exercícios realizados, esse item se encaixa em estudos de comparações de tratamento e de tipo Placebo. No critério 7 leva em consideração se os avaliadores avaliaram de forma cega todas as variáveis de estudo. $\mathrm{O}$ item 8 se trata de resultado do estado os participantes são divididos em grupos, sendo realizados diferentes exercícios, obtendo um resultado chave após algumas semanas, e se ouvi perdas amostrais ao decorrer do estudo. O critério 9 trata-se da utilização da análise por intenção de tratamento, para que assim todos os sujeitos precisam ser analisados no fim do estudo, mesmo que não tenha encerrado o tratamento ou escolheu mudar de grupo ou intervenção. Já o critério 10 quer saber se ouve comparações dês estatísticas entre os grupos considerando os tipos de tratamento, para obter um resultado chave. Por fim o item 11 se refere a medidas de precisão e variabilidade obtendo ao menos uma palavra chave, com isso o item 11 implica no efeito de intervenção e diferentes resultados nos grupos trabalhados. A escala PEDro terá sua avaliação de 0 a 10 pontos, sendo que o critério 1 não é considerado ponto somatório.

\section{Resultados e Discussão}

Objetivo do presente estudo foi determinar se o fortalecimento muscular é eficaz em Tendinopatia do manguito rotador, para tanto localizou-se sete artigos, quanto as características do estudo encontrado observou-se que a amostra variou de 20 indivíduos até 120 indivíduos conforme está na Tabela 1, ao observar os grupos de tratamento notou-se que pelo menos um grupo teve fortalecimento muscular, os estudos tiveram de dois a três grupos de pesquisa, e além do fortalecimento muscular as técnicas empregadas foram alongamentos, terapia manual, movimento ativo assistido e treinamento muscular, a menor frequência observada foi de uma semana a vinte e duas semanas de duas a três vezes por dia. Os resultados e conclusões dos estudos são observados na Tabela 2. 
Tabela 1. Características do Estudo.

\begin{tabular}{|c|c|c|c|c|}
\hline Estudo & Amostra & Grupos de pesquisa & Frequência de tratamento & Método de análise \\
\hline Heron et al., (2017) & 120 & $\begin{array}{l}\text { ROM: exercícios minimamente } \\
\text { carregado/ grupo cadeia cinética } \\
\text { aberta/ grupo cadeia cinética } \\
\text { fechada / }\end{array}$ & $\begin{array}{l}\text { Três séries de } 10 \text { repetições, } \\
\text { duas vezes por dia }\end{array}$ & $\begin{array}{l}\text { Índice de Incapacidade e } \\
\text { Dor no Ombro (SPADI) }\end{array}$ \\
\hline Dejaco et al., (2016) & 41 & $\begin{array}{l}\text { EE: Exercício excêntrico GC: grupo } \\
\text { controle }\end{array}$ & $\begin{array}{l}\text { Oito exercícios diferentes por } \\
\text { dia durante } 12 \text { semanas }\end{array}$ & $\begin{array}{l}\text { Goniômetro, amplitude, } \\
\text { dor através da escala de } \\
\text { vaz }\end{array}$ \\
\hline $\begin{array}{l}\text { Broudeau et al., } \\
(2019)\end{array}$ & 42 & $\begin{array}{l}\text { Dois estavam no grupo RCEx } \\
\text { enquanto o outro estava no grupo } \\
\text { de coativação RCEx }+ \text {. }\end{array}$ & 6 semanas & $\begin{array}{l}\text { Os dados foram analisados } \\
\text { usando ANOVAs de } \\
\text { modelo misto. }\end{array}$ \\
\hline Dupuis et al., (2018) & 48 & $\begin{array}{l}\text { Grupo I: o grupo experimental que } \\
\text { realizou exercícios isométricos; e } \\
\text { grupo II: o grupo controle que } \\
\text { recebeu orientação para descansar } \\
\text { de atividades agravantes e foi } \\
\text { orientado a aplicar envoltórios de } \\
\text { gelo. }\end{array}$ & $\begin{array}{l}\text { Os participantes receberam } \\
\text { um guia para a porcentagem } \\
\text { de força máxima, número de } \\
\text { repetições }(3-4) \text { e número de } \\
\text { segundos agarres (20-30 s), } \\
\text { que progrediu do dia } 1 \text { para o } \\
\text { dia } 14 \text {. Um nível máximo de } \\
\text { dor de } 5 / 10 \text { foi aceito durante } \\
\text { a realização das contrações, } \\
\text { desde que estabilizasse } 10-15 \\
\text { minutos após os exercícios. }\end{array}$ & $\begin{array}{l}\text { Educação sobre postura e } \\
\text { posicionamento do ombro } \\
\text { durante as atividades } \\
\text { diárias. Exercícios de } \\
\text { abdução e rotação lateral } \\
\text { isométrica a ser realizado } \\
\text { três vezes ao dia durante } \\
14 \text { dias. Fortalecimento. } \\
\text { Exercícios de rotação } \\
\text { externa e interna de ombro } \\
\text { isotônico resistido }\end{array}$ \\
\hline Belley et al., (2018) & 40 & $\begin{array}{l}\text { Um recebendo um programa de } \\
\text { reabilitação centrado no } \\
\text { treinamento sensório-motor com a- } \\
\text { tDCS e outro recebendo o mesmo } \\
\text { programa de reabilitação com sham } \\
\text { a-tDCS }\end{array}$ & $\begin{array}{l}\text { Cada sessão teve duração de } \\
40 \text { minutos, treinamento } \\
\text { sensório-motor e o equilíbrio } \\
\text { do tempo consistiu e revisão } \\
\text { de exercícios domiciliares e } \\
\text { educação sobre tendinopatia } \\
\text { do manguito rotador. } \\
3 \text { séries de } 10 \text { repetições sem } \\
\text { dor ou fadiga }\end{array}$ & $\begin{array}{l}\text { Os exercícios de controle } \\
\text { de ombro durante a } \\
\text { elevação do braço } \\
\text { Fortalecimento, } \\
\text { alongamento e a terapia } \\
\text { manual foram realizados } \\
\text { se necessário }\end{array}$ \\
\hline Salvador et al., (2020) & 26 & $\begin{array}{l}\text { GRUPO EXPERIMENTAL; } \\
\text { GRUPO CONTROLE }\end{array}$ & $\begin{array}{l}\text { Contagem de } 15-20 \mathrm{~s} \text {, três } \\
\text { séries de } 12 \text { repetições por } \\
\text { grupo muscular (a maioria } \\
\text { dos programas de } \\
\text { fortalecimento inclui } 2-3 \\
\text { séries de } 8-20 \text { repetições, } \\
\text { decidimos como um } \\
\text { programa regular incluir uma } \\
\text { das } 3 \text { séries de } 12 \text { repetições }\end{array}$ & $\begin{array}{l}\text { Amplitude de movimento } \\
\text { assistida ativa para o } \\
\text { ombro afetado com um } \\
\text { ganho de mobilidade } \\
\text { progressivo, conforme } \\
\text { tolerado.Alongamento } \\
\text { muscular assistido para } \\
\text { trapézio superior, peitoral, } \\
\text { deltóide, cápsula articular } \\
\text { anterior e posterior do } \\
\text { ombro, grande dorsal, } \\
\text { músculos rotadores, } \\
\text { bíceps e tríceps. }\end{array}$ \\
\hline Ager et al., (2018) & $\begin{array}{l}\text { O } \\
\text { tamanho } \\
\text { do grupo } \\
\text { variou } \\
\text { entre } 5 \text { e } \\
20 \\
\text { participant } \\
\text { es }\end{array}$ & $\begin{array}{l}\text { Cada participante designado para o } \\
\text { grupo UpEx-NTP. }\end{array}$ & 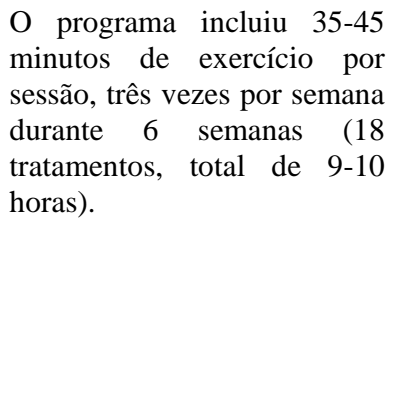 & $\begin{array}{lr}\text { Em um } & \text { consenso } \\
\text { neuromuscular } & \\
\text { supervisionado em } & \text { grupo } \\
\text { de multiestações de } & 6 \\
\text { semanas apoiou uma } & \\
\text { abordagem de reabilitação } \\
\text { de exercício ativo que } \\
\text { incluiu exercícios de } \\
\text { fortalecimento } \\
\text { treinamento } \\
\text { neuromuscular. }\end{array}$ \\
\hline
\end{tabular}

Fonte: Henrique et al (2021).

Em relação aos resultados observou-se que os estudos no qual apresentaram significância foram sete artigos sendo que não houve diferença entre os grupos, mas ouve melhora nos sintomas e no quadro álgico de todos os grupos, no entanto não 
obteve melhoras a longo prazo, a conclusão foi que os exercícios de fortalecimento muscular em cadeia aberta, fechada, exercícios isométricos e exercícios de amplitude de movimento tiveram benefícios na dor e na função de cada indivíduo, após 12 semanas, observasse na Tabela 2.

Tabela 2. Resultados e conclusões dos estudos selecionados.

\begin{tabular}{ll}
\hline & Resultados \\
\hline Eron et al. (2017) & Todos os três programas resultaram em diminuições \\
& significativas na pontuação SPADI, porém não houve \\
& diferenças significativas entre os grupos. Os participantes que \\
& fizeram um MCIC nos sintomas foram semelhantes em todos os \\
& grupos, no entanto, mais participantes se deterioraram no grupo \\
& ROM. A taxa de abandono foi maior no grupo CC, mas quando \\
& apenas os pacientes que completaram o tratamento foram \\
& considerados, mais pacientes no grupo CC reduziram \\
& significativamente a dor e a incapacidade.
\end{tabular}

Dejaco et al. (2017) Após 26 semanas, ambos os grupos mostraram um aumento significativo no escore de Constant Murley e uma diminuição significativa nos escores VAS. Nenhuma diferença foi encontrada entre os grupos, para qualquer uma das medidas de desfecho avaliadas.

Dupuis et al. (2018) Após as intervenções, ambos os grupos mostraram melhorias estatisticamente significativas nos sintomas e na função na semana 2 e na semana 6 em comparação com a linha de base. No entanto, não houve interação significativa grupo $\times$ tempo. Não houve efeito do tempo na distância acromioumeral, força do ombro e amplitude de movimento ativa, bem como nenhuma interação tempo $\times$ grupo.

Belley et al. (2018) Ambos os grupos mostraram melhora estatisticamente significativa no DASH e 20 WORC em 3, 6 e 12 semanas e em em $45^{\circ}$ e $60^{\circ} \mathrm{em} 6$ semanas $(\mathrm{P}<0,05)$. Insignificante

21 A interação Grupo por Tempo foi observada para todos os resultados $(\mathrm{P}>0,43)$

Ager et al. (2018)

Nenhum grupo significativo ( $\mathrm{p} \geq 0,16$ ) ou interações grupo $\times$ tempo $(p \geq 0,11)$ foram encontrados para as análises ITT ou por protocolo. Foi estabelecido um efeito de tempo estatisticamente significativo ( $p<0,001$ ) para o DASH e WORC, mostrando que ambos os grupos melhoraram com o tempo.

Boudreau et al. (2019) Não foi observada interação grupo por tempo significativa para o questionário Deficiências do braço, ombro e mão, índice Western Ontario Rotator Cuff, escala visual analógica e distância acromio-umeral $\quad(\mathrm{P} \geq 0,055)$. Efeitos de tempo significativos foram obtidos para o índice Western Ontario Rotator Cuff e escala visual analógica para dor com movimento $(\mathrm{P}<0,001)$.

Salvador et al. (2020) Encontramos diferenças intergrupos na escala de Constant em 1 e 3 meses $(p<0,05)$ e na força nos meses 1 e 3
Conclusão

Os exercícios de cadeia aberta, cadeia fechada e amplitude de movimento parecem ser eficazes em provocar alterações de curto prazo na dor e na incapacidade em pacientes com tendinopatia do manguito rotador.

Um programa de treinamento excêntrico isolado de 12 semanas do manguito rotador é benéfico para a função do ombro e dor após 26 semanas em pacientes com tendinopatia do manguito rotador. No entanto, não é mais benéfico do que um programa de exercícios convencional para o manguito rotador e os músculos escapulares.

Os resultados mostraram uma melhora estatisticamente significativa nos sintomas e função em ambos os grupos, mas não houve diferença entre o efeito de curto prazo de uma abordagem baseada em crioterapia e um programa de exercícios de recarga gradual.

Os resultados não demonstram quaisquer efeitos adicionais de a-tDCS durante um programa de reabilitação em indivíduos com tendinopatia $\mathrm{CR}$.

Nossos dados preliminares demonstram que ambas as abordagens de reabilitação, baseadas em exercícios ativos, não foram estatisticamente diferentes entre si e derivaram benefícios semelhantes ao longo do tempo para uma população militar.

Os presentes achados mostram que a adição da coativação de adutores glenoumerais a um programa de fortalecimento do manguito rotador não resulta em melhora da eficácia a curto prazo em nenhum dos resultados medidos.

O fortalecimento excêntrico e concêntrico foram bem tolerados; ambos mostram melhora precoce 
( $p<0,05)$. Observamos diferenças estruturais no tendão (cicatrização) entre os grupos aos 3 e 12 meses. da dor, funcionalidade e estrutura do tendão. $\mathrm{O}$ treinamento excêntrico parece ser mais eficaz do que o concêntrico na melhora inicial da funcionalidade, força e cicatrização do tendão.

Fonte: Henrique et al (2021).

O item um não conta pontuação, o autor Eron et al, (2017) não se enquadrou ao item 5 que compreende que todos os participantes alocados de forma cega, o autor Eron et al, (2017) não se encaixou ao item 6 que compreende em todos os fisioterapeutas fizeram de forma cega as terapia sãos pacientes, Eron et al, (2017) não se encaixou ao item 7 compreende em todos os avaliadores fizeram de forma cega, os autores Djaco et al, (2017) e Broudeau et al 2019 não se encaixam ao item 8 pelo menos 85\% foram acompanhados apropriadamente, o artigo do autor Broudeau et al, (2019) não se encaixou ao item 9 analise com intuito de tratamento , o artigo de Dejaco et al, (2017) não se encaixou ao item 11 proporcionar analises de tratamento, os demais itens foram compridos por todos os estudos, observasse na tabela 3.

Tabela 3. Pontuação da escala Pedro para os artigos selecionados.

Critérios da Escala Pedro para os artigos selecionados

\begin{tabular}{|c|c|c|c|c|c|c|c|c|c|c|}
\hline & Item 2 & Item 3 & Item 4 & Item 5 & Item 6 & Item 7 & Item 8 & Item 9 & Item 10 & Item 11 \\
\hline Eron et al. (2017) & $\mathrm{X}$ & $\mathrm{X}$ & $\mathrm{X}$ & 0 & 0 & 0 & $\mathrm{X}$ & $\mathrm{X}$ & $\mathrm{X}$ & $\mathrm{X}$ \\
\hline Dejaco et al. (2017) & $\mathrm{X}$ & $\mathrm{X}$ & $\mathrm{X}$ & $\mathrm{X}$ & $\mathrm{X}$ & $\mathrm{X}$ & 0 & $\mathrm{X}$ & $\mathrm{X}$ & 0 \\
\hline Dupuis et al. (2018) & $\mathrm{X}$ & $\mathrm{X}$ & $\mathrm{X}$ & $\mathrm{X}$ & $\mathrm{X}$ & $\mathrm{X}$ & $\mathrm{X}$ & $\mathrm{X}$ & $\mathrm{X}$ & $\mathrm{X}$ \\
\hline Belley et al. (2018) & $\mathrm{X}$ & $\mathrm{X}$ & $\mathrm{X}$ & $\mathrm{X}$ & $\mathrm{X}$ & $\mathrm{X}$ & $\mathrm{X}$ & $\mathrm{X}$ & $\mathrm{X}$ & $\mathrm{X}$ \\
\hline Ager et al. (2018) & $\mathrm{X}$ & $\mathrm{X}$ & $\mathrm{X}$ & $\mathrm{X}$ & $\mathrm{X}$ & $\mathrm{X}$ & $\mathrm{X}$ & $\mathrm{X}$ & $\mathrm{X}$ & $\mathrm{X}$ \\
\hline Boudreau et al. (2019) & $\mathrm{X}$ & $\mathrm{X}$ & $\mathrm{X}$ & $\mathrm{X}$ & $\mathrm{X}$ & $\mathrm{X}$ & 0 & 0 & $\mathrm{X}$ & $\mathrm{X}$ \\
\hline Salvador et al. (2020) & $X$ & $X$ & $X$ & $X$ & $X$ & $X$ & $X$ & $X$ & $X$ & $X$ \\
\hline
\end{tabular}

(2): Alocação randomizada; (3):Atribuição mascarada; (4): Similaridade no início do tratamento; (5): assuntos cegos; (6): Fisioterapeutas cegos; (7): avaliadores cegos; (8): acompanhamento apropriado; (9): análise por intuito de tratar; (10): correlações intergrupos; (11): uso de medidas de precisão e variabilidade.

Fonte: Henrique et al (2021).

O fluxograma presente na figura 1, ira amostrar quantos artigos foram achados, quais foram eliminados e o porquê de ser eliminado, sendo que para elaboração do presente estudo foram selecionados apenas 7 artigos, segundo os critérios de inclusão e exclusão. 
Figura 1: Fluxograma.

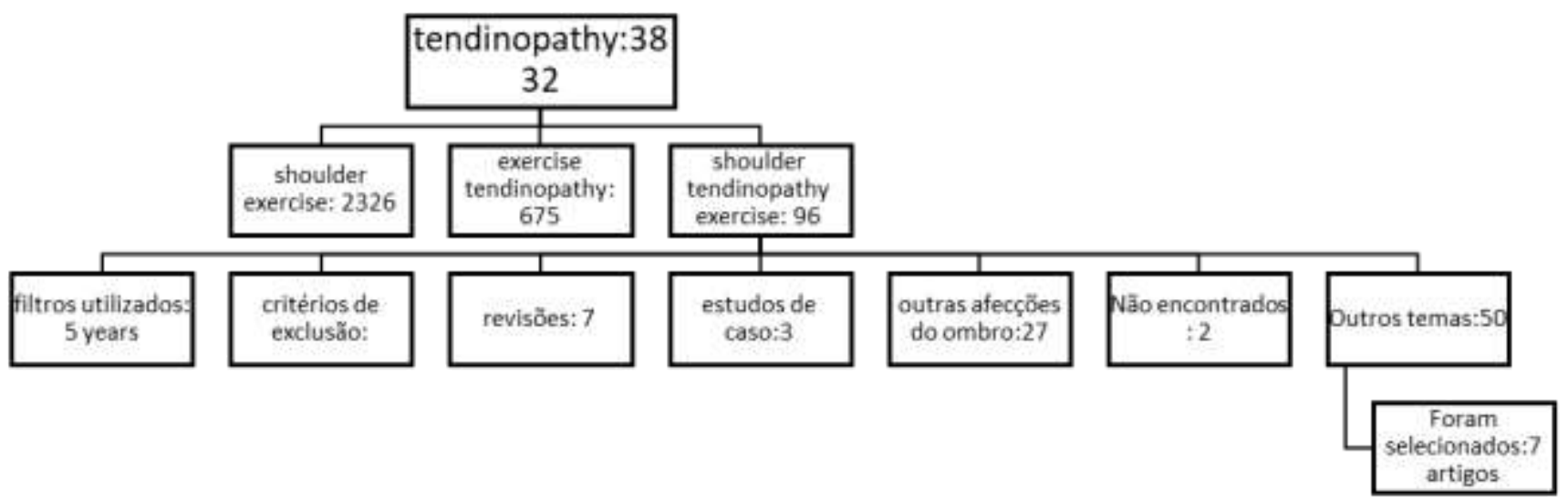

Fonte: Henrique et al (2021).

A dor do ombro é a maior causa de busca por fisioterapeutas e médicos. A Tendinopatia do Manguito Rotador (RC), é uma lesão nos tendões do supra espinhoso, infra espinhoso, redondo menor e subescapular que geralmente é causada por movimentos repetitivos, que são altamente prevalentes, em alguns estudos se tem demonstrado a eficácia em melhoras utilizando de exercícios de fortalecimento muscular, concêntrico, excêntrico e isométrico.

Kooijman et al., (2013) retatra em seu artigo que "A diretriz para queixas de ombro do Dutch College of General Practitioners recomenda uma abordagem gradual na qual o paciente é encaminhado para fisioterapia quando não há melhora com repouso e aconselhamento ('espere para ver') e analgésicos por uma ou duas semanas (de preferência paracetamol). Quando a dor é o principal problema, o tratamento prolongado com analgesia é indicado. A fisioterapia é indicada principalmente na presença de amplitude de movimento limitada ou outras limitações funcionais".

De acordo com o autor Parle et al., (2016) "O tratamento precoce da tendinopatia aguda do manguito rotador envolve educação do paciente e repouso relativo, e pode incluir medicamentos antiinflamatórios não esteroidais (AINEs) par reduzir a dor, o inchaço e a inflamação. As injeções subacromiais de corticosteroides também são usadas para atingir o mesmo objetivo."

Segundo Heron et al., (2017) "os exercícios de cadeia aberta resistido (OC), fechada resistido (CC) e os movimentos de amplitude de movimento (ROM) são eficazes para o tratamento da dor, funcionalidade do indivíduo", as exercícios OC executados por cada participante foi o movimento de rotações e abdução de ombro com a resistência da faixa elástica com series de 10 vezes, conforme os participantes fossem progredindo sem dor ocorreria o aumento das cargas, exercícios CC os movimentos realizados foram de colocar as mãos na cadeira e tentar levantar o corpo da cadeira com o braço saldável e depois com o afetado e foram progredindo conforme não sentiam dor, ROM faziam movimentos com o bastão para melhorar a Amplitude de movimento (ADM). Conforme Dejaco et al, (2017) "os exercícios excêntricos (EE) isolados sendo que tem uma eficácia por volta de 26 semanas no quadro álgico no tratamento do manguito rotador, mais não apresentou benefícios maior do que um exercício convencional para manguito rotado" o grupo EE foi dividido em dois exercícios para o manguito rotador, um com auxílio de uma faixa elástica, em decúbito dorsal, sendo de 6 a 8 repetições do exercício, o segundo exercício era realizado uma abdução de lata vazia até um limiar de dor de 5 na escala EVA, todos os participantes realizaram exercícios de alongamento no grupo EE, o grupo CG realizou exercícios de fortalecimento com movimento de abdução com resistência de 
alteres, rotações interna e externas com a faixa elástica, alongamentos. Nenhum dos exercício se sobressaiu ao outro, todos aliviaram a dor e melhoram a função dos participantes.

De acordo com o autor Dupuis et al., (2018) "não houve interação com o tempo significativo, no entanto em seus resultados ficou comprovado uma melhora significativa nos sintomas e funcionalidade do participante da pesquisa, não houve diferença entre a abordagem com crioterapia e exercícios de cargas graduais”, a crioterapia ajudou a reduzir o edema e a dor no membro afetado, os participantes do grupo do descanso foram orientados a colocar gelo durante 15 minutos, 3 vezes ao dia, foram orientados a descansar e não fazer movimentos que possa sentir dor no local, o grupo de carregamento gradual os participantes foram instruídos a fazer exercícios de abdução, e rotações laterais com resistência do membro não afetado, não teve um estudo que se sobressaiu ao outro, ambos tiveram melhora nos movimentos e no quadro álgico. $\mathrm{O}$ autor Belley et al. (2018) discorre sobre "Ambos os grupos mostraram melhora estatisticamente em 3, 6 e 12 semanas e em AHD em $45^{\circ}$ e $60^{\circ}$ em 6 semanas. Nenhuma interação Grupo por Tempo significativa foi observada para todos os resultados", treinamento sensorial e motor foram realizados exercícios de fortalecimento muscular com elevação do membro afetado com aumento gradual da resistência desde movimentos passivos a ativos, foi repassado exercícios para serem feitos em casa como um reforço, todos os participantes tiveram acompanhamento de qualidade, durante o estudo e não houve interação de melhoras com o tempo. Desse modo Ager et al. (2018) relatou que "o presente estudo observou que ocorreu uma melhora sobre o efeito de tempo para o DASH e WORC, na abordagem terapêutica com exercícios ativos tiveram melhoras significativas" o DASH é um questionário utilizado para avaliar os sintomas e a capacidade de cada participante da pesquisa para realizar um determinado movimento, WORC é um questionário especifico para lesões no RC.

Para o autor Ingwersen et al., (2017) a "Tendinopatia do manguito rotador, exercícios excêntricos com carga têm se mostrado superiores aos exercícios sem carga e igual ao treinamento tradicional do manguito rotador na redução da dor e melhoria da função. A superioridade do exercício progressivo de alta carga foi demonstrada apenas quando comparada com controles passivos". Boudreau et al., (2019) relata que "os resultados mostram que a adição da ativação de adutores de fortalecimento do manguito rotador obteve melhora curto prazo em nenhum dos resultados do estudo" foram utilizados exercícios de fortalecimento nos mesmos grupos, sendo que quando. Salvador et al, (2020) relata que "os exercícios excêntricos e concêntricos foram bem tolerados pelos participantes, no protocolo tiveram o mesmo percentual significativo, pois, melhorou precocemente a dor e a funcionalidade de cada paciente". O exercício excêntricos realizados com auxílio de pesos e foi ensinado como o participante poderia fazer, se sentir dor para o exercício. O exercício Concêntrico foi realizado pelo grupo controle os participantes foram instruído a realizar movimentos articulares, os pacientes em ambos exercícios utilizaram de pesos variados conforme ao seu quadro álgico.

\section{Considerações Finais}

O presente estudo de revisão bibliográfica verificou a eficácia do fortalecimento muscular na Tendinopatia RC, em todos os estudos inclusos, os exercícios de FM excêntricos e alongamentos obtiveram grandes resultados, desde o período do quadro álgico até a funcionalidade e total mobilidade de cada participante, ou seja quando se trata de intervenções no RC exercícios de fortalecimento variando do tempo de tratamento e intensidade, será alcançado os objetivos de cura da doença, porém é de sumo importância obter novos estudos afim de traçar novos meios de tratamento para tendinopatia no manguito rotador.

\section{Agradecimentos}

Agradecemos a todos que direta ou indiretamente contribuíram para a realização e sucesso do artigo. 


\section{Referências}

Ager, A. L., Roy, J.-S., Gamache, F., \& Hébert, L. J. (2018). The Effectiveness of an Upper Extremity Neuromuscular Training Program on the Shoulder Function of Military Members With a Rotator Cuff Tendinopathy: A Pilot Randomized Controlled Trial. Military Medicine, 184(5-6), e385-e393. https://doi.org/10.1093/milmed/usy294

Belley, A. F.; Mercier, C.; Bastien, M.; Léonard, G.; Gaudreault, N. \& Roy, J. S. (2018). Anodal Transcranial Direct-Current Stimulation to Enhance Rehabilitation in Individuals With Rotator Cuff Tendinopathy: A Triple-Blind Randomized Controlled Trial. Journal of Orthopaedic \& Sports Physical Therapy, 48(7), 541-551. https://doi.org/10.2519/jospt.2018.7871

Boudreau, N., Gaudreault, N., Roy, J.-S., Bédard, S., \& Balg, F. (2019). The Addition of Glenohumeral Adductor Coactivation to a Rotator Cuff Exercise Program for Rotator Cuff Tendinopathy: A Single-Blind Randomized Controlled Trial. Journal of Orthopaedic \& Sports Physical Therapy, 49(3), 126-135. https://doi.org/10.2519/jospt.2019.8240

Dejaco, B., Habets, B., van Loon, C., van Grinsven, S., \& van Cingel, R. (2016). Eccentric versus conventional exercise therapy in patients with rotator cuff tendinopathy: a randomized, single blinded, clinical trial. Knee Surgery, Sports Traumatology, Arthroscopy, 25(7), 20512059. https://doi.org/10.1007/s00167-016-4223-x

Dupuis, F., Barrett, E., Dubé, M.-O., McCreesh, K. M., Lewis, J. S., \& Roy, J.-S. (2018). Cryotherapy or gradual reloading exercises in acute presentations of rotator cuff tendinopathy: a randomised controlled trial. BMJ Open Sport \& Exercise Medicine,4(1), e000477. https://doi.org/10.1136/bmjsem-2018-000477

Escala PEDro. (n.d.). PEDro. Retrieved October 25, 2021, from https://pedro.org.au/portuguese/resources/pedro-scale/

Heron, S. R., Woby, S. R., \& Thompson, D. P. (2017). Comparison of three types of exercise in the treatment of rotator cuff tendinopathy/shoulder impingement syndrome: A randomized controlled trial. Physiotherapy, 103(2), 167-173. https://doi.org/10.1016/j.physio.2016.09.001

Ingwersen, K. G., Jensen, S. L., Sørensen, L., Jørgensen, H. R., Christensen, R., Søgaard, K., \& Juul-Kristensen, B. (2017). Three Months of Progressive High-Load Versus Traditional Low-Load Strength Training Among Patients With Rotator Cuff Tendinopathy: Primary Results From the Double-Blind Randomized Controlled RoCTEx Trial. Orthopaedic Journal of Sports Medicine, 5(8), 232596711772329. https://doi.org/10.1177/2325967117723292

Kooijman, M., Swinkels, I., Dijk, C. van, Bakker, D. de, \& Veenhof, C. (2013). Patients with shoulder syndromes in general and physiotherapy practice: an observational study. BMC Musculoskeletal Disorders, 14(1). https://doi.org/10.1186/1471-2474-14-128

Lewis J, Dor no ombro relacionada ao manguito rotador: Avaliação, manejo e incertezas, Terapia manual (2016), doi: 10.1016 / j.math.2016.03.009.

Lewis, J. S. (2009). Rotator cuff tendinopathy. British Journal of Sports Medicine,43(4), 236-241. https://doi.org/10.1136/bjsm.2008.052175

Lewis, J. S. (2009). Rotator cuff tendinopathy/subacromial impingement syndrome: is it time for a new method of assessment? British Journal of Sports Medicine, 43(4), 259-264. https://doi.org/10.1136/bjsm.2008.052183

Littlewood, C., Ashton, J., Chance-Larsen, K., May, S., \& Sturrock, B. (2012). Exercise for rotator cuff tendinopathy: a systematic review. Physiotherapy, 98(2), 101-109. https://doi.org/10.1016/j.physio.2011.08.002

Longo, U. G., Berton, A., Papapietro, N., Maffulli, N., \& Denaro, V. (2011). Epidemiology, Genetics and Biological Factors of Rotator Cuff Tears. Rotator Cuff Tear, 1-9. https://doi.org/10.1159/000328868

Macias-Hernandez, S. I.; Garcia-Morales, J. R.; Hernandez-Diaz, C.; Tapia-Ferrusco, I.. Velez-Gutierrez, O. B. \& Nava-Bringas, T. I. (2020). Tolerance and effectiveness of eccentric vs. concentric muscle strengthening in rotator cuff partial tears and moderate to severe shoulder pain. A randomized pilot study. (2021). Journal of Clinical Orthopaedics and Trauma, 14, 106-112. https://doi.org/10.1016/j.jcot.2020.07.031

Moher, D., Liberati, A., Tetzlaff, J., \& Altman, D. G. (2009). Preferred Reporting Items for Systematic Reviews and Meta-Analyses: the PRISMA Statement. PLoS Medicine, 6(7), e1000097. https://doi.org/10.1371/journal.pmed.1000097

Neer CS II. Acromioplastia anterior para síndrome do impacto crônicono ombro: um relato preliminar.J Bone Joint Surg Am. 1972; 54 (1):41-50.

Nirschl, R. P. (1969). Mesenchymal syndrome. Virginia Medical Monthly, 96(11), 659-662. https://pubmed.ncbi.nlm.nih.gov/5377568/

Parle, P. J., Riddiford-Harland, D. L., Howitt, C. D., \& Lewis, J. S. (2016). Acute rotator cuff tendinopathy: does ice, low load isometric exercise, or a combination of the two produce an analgaesic effect? British Journal of Sports Medicine, 51(3), 208-209.

https://doi.org/10.1136/bjsports-2016-096107, 
Research, Society and Development, v. 10, n. 14, e524101422298, 2021

(CC BY 4.0) | ISSN 2525-3409 | DOI: http://dx.doi.org/10.33448/rsd-v10i14.22298

Titchener, A. G., White, J. J. E., Hinchliffe, S. R., Tambe, A. A., Hubbard, R. B., \& Clark, D. I. (2014). Comorbidities in rotator cuff disease: a case-control study. Journal of Shoulder and Elbow Surgery, 23(9), 1282-1288. https://doi.org/10.1016/j.jse.2013.12.019 